\section{$\underset{\substack{\text { hommes } \\ \text { \& migrations }}}{ }$}

\section{Hommes \& migrations}

Revue française de référence sur les dynamiques

migratoires

1307 | 2014

L'Afrique qualifiée dans la mondialisation

\title{
Bandjoun Station
}

Entretien avec son fondateur, l'artiste plasticien Barthélémy Toguo

\section{Laure Poinsot}

\section{OpenEdition}

1 Journals

\section{Édition électronique}

URL : http://journals.openedition.org/hommesmigrations/2889

DOI : 10.4000/hommesmigrations.2889

ISSN : 2262-3353

Éditeur

Musée national de l'histoire de l'immigration

\section{Édition imprimée}

Date de publication : 1 juillet 2014

Pagination : 116-120

ISBN : 978-2-919040-28-5

ISSN : 1142-852X

Référence électronique

Laure Poinsot, « Bandjoun Station », Hommes \& migrations [En ligne], 1307 | 2014, mis en ligne le 01 juillet 2017, consulté le 01 mai 2019. URL : http://journals.openedition.org/hommesmigrations/2889 ; DOI : 10.4000/hommesmigrations.2889 


\section{BANDJOUN STATION ENTRETIEN AVEC SON FONDATEUR, L'ARTISTE PLASTICIEN BARTHÉLÉMY TOGUO}

réalisé par LAURE POINSOT, réalisatrice de documentaire.

\begin{abstract}
Hommes \& Migrations : Vous êtes artiste plasticien. Vous venez d'arriver à Bandjoun (Cameroun) depuis Paris où vous vous vivez et où vous avez votre atelier. Comment vous partagez-vous entre ces deux lieux?

Barthélémy Toguo : J'essaye de bien morganiser. À Bandjoun, je peux réaliser des œuvres monumentales que j'ai pensées et conçues à Paris, car au Cameroun, j'ai de l'espace et des assistants. Paris pour moi est devenu davantage un lieu de laboratoire et de conception que de production. Je suis un homme de mon temps qui profite des moyens de communication performants d'aujourd'hui pour voyager et produire. Par exemple, de Paris ou de Bandjoun, je peux envoyer par mail le processus de fabrication d'une œuvre qui sera exposée à New York ou à Pékin, produire des œuvres à distance, ou dans n'importe quel atelier de mon choix. Et puis des choses se créent dans le voyage. Comme le dit si bien Paulo Coelho, quand on va vers les choses, les choses viennent vers nous. C'est tout ce foisonnement qui m'inspire et me donne de nouveaux sujets de travail.
\end{abstract}

$H \& M$ : Cet entretien est publié dans un numéro de la revue Hommes \& Migrations sur les diasporas africaines. Vous sentez-vous appartenir à une diaspora ? Qu'est-ce que cela signifie pour vous? Qu'est-ce que cela implique pour vous en terme de références, d'identité ?

B. T. : Je fais partie d'un groupe, d'une communauté, la communauté noire africaine, d'une ethnie, d'une culture, donc d'une diaspora dans la mesure où depuis près de vingt-cinq ans, je vis principalement à létranger. J'ai étudié et j'ai commencé ma carrière professionnelle hors du Cameroun. En tant que ressortissant de ce pays, j'ai un devoir à assumer, je me dois de contribuer à son développement. Car le fait de voyager, d'être en contact avec des cultures différentes de la mienne, m'enrichit, m'ouvre l'esprit. C'est ce que j'ai envie de partager avec mes compatriotes restés au pays, pour contribuer au changement des mentalités, au développement culturel et artistique du Cameroun. Je parle en tant qu'Africain et ressortissant de cette communauté d"intellectuels". Nous devons tous apporter notre pierre à l'édifice et au développement de ce continent. Et si tous les Africains de cette diaspora pouvaient apporter une contribution, je crois que l'Afrique se développerait mieux dans les domaines agricole, éducatif, sportif, économique, sanitaire. Dans mon cas, je me suis dit qu'en édifiant un centre d'art, je donnerai aux jeunes artistes l'occasion de produire et de montrer le résultat de leur travail à un public local, national, voire international. 
$H \& M$ : Quand on arrive à Bandjoun Station, on est frappé par cette architecture inhabituelle avec de gigantesques mosaïques colorées sur les murs. Comment avez-vous pensé et réalisé cette construction?

B. T. : Cette architecture a été construite par la population de Bandjoun, par des maçons et des artisans d'ici. J'ai senti qu'ils étaient heureux de participer à l'édification d'une si belle architecture. Maintenant, elle fait la fierté du village tout entier. Le pignon de la toiture de Bandjoun Station s'élève à 11 mètres et une sculpture mappemonde comme un chapeau la coiffe à 13 mètres. Le côté graphique est très fort aussi, les couleurs, les dessins, les mosaïques ornent joliment la façade. À chaque fois qu'il y a une fête religieuse ou nationale, tout le village vient poser devant les fresques de Banjoun Station. Tout le monde revêt sa plus belle tenue et vient ajouter sa présence colorée.

\section{$H \& M$ : Pourquoi avez-vous appelé ce lieu "Bandjoun Station"?}

B. T. : D'abord simplement parce que le lieu se trouve à Bandjoun et ensuite parce que Station signifie "arrêt" en anglais. Cet arrêt est un appelà la rencontre, à la découverte, au partage de cultures et d'univers différents avant de prendre un nouveau départ.

\section{H\&M: Comment avez-vous imaginé, rêvé} ce lieu?

B. T. : J'avais au départ pensé à créer une école, un institut d'arts visuels. Mais mettre sur pied une école est un processus fastidieux et compliqué. J'ai jugé préférable de créer un lieu où les choses se font, se façonnent, se créent, un espace où les projets en adéquation avec l'environnement humain et naturel se réalisent. À Bandjoun Station, tout artiste autodidacte ou académique peut être accueilli en résidence et produire des œuvres. Bien que j'aie moi-même suivi un cursus universitaire artistique, je sais qu'on peut être artiste sans passer par cette formation, pourvu que l'on soit dans l'élan de la création. Dès qu'on est dans cet élan, on est accepté, que l'on soit diplômé ou autodidacte : on est tous des confrères artistes.

$H \& M$ : Pourquoi avez-vous installé ce lieu de création à Bandjoun ?

B. T. : D'abord parce que c'est le village de mes ancêtres, de mes parents. Au-delà de cet aspect personnel, Bandjoun a été un grand pôle artistique au cours des siècles passés. Le premier chasseur qui s'y est installé était un artiste forgeron qui produisait des outils pour la chasse. Il avait quitté la famille royale de Baleng pour aller conquérir de nouvelles terres. Pour constituer sa population, il a décidé d'acheter des gens rejetés par d'autres communautés. C'est de là que vient le nom Bandjoun : "les gens qui achètent". Je n'ai donc pas choisi Bandjoun par hasard.
Vue extérieure de la Bandjoun Station, Bandjoun (Cameroun). (c) D.R.

Dans cette région, il existe encore une pratique artistique selon des traditions séculaires transmises de génération en génération dans la forge, la poterie, la sculpture, l'architecture. L'art y est 
omniprésent, aussi bien sur les pagnes colorés des femmes qu'à travers les architectures des maisons à toit conique ou les motifs des murs de certaines cases traditionnelles.

Mon idée avec Bandjoun Station était de marier l'art classique africain et l'art contemporain mondial, d'exposer ces œuvres dans un même espace, sans ghettoïsation ou hiérarchie de valeurs. C'est pourquoi l'une des missions de Bandjoun Station est d'aller à la découverte de ce qui a été fait tout en montrant ce qui existe et se fait à présent. C'est ainsi que Bandjoun Station deviendra un carrefour, un véritable lieu de rencontre entre l'art classique et l'art contemporain.

\section{$H \& M$ : Vous avez installé dans le hall d'accueil une de vos œuvres majeures The New World Climax. Pourquoi avoir choisi d'installer cette œuvre-là plus qu'une autre ?}

B. T. : D'abord parce qu'elle occupe du volume et que je voulais à l'entrée une œuvre monumentale, une présence physique. Et aussi parce que ces tampons géants regroupent tout un ensemble de problématiques contemporaines sur l'exil, le voyage, la discrimination, les problèmes économiques, le terrorisme, les frontières, l'holocauste, la sexualité, le mariage gay, l'exploitation, la torture, la suprématie, l'envie d'écraser l'autre, tout est là. Je viens de faire un tampon au Canada sur l'enlèvement des lycéennes nigérianes par Boko Aram, "Bring back our girls".

$H \& M$ : Ces questions de discrimination et d'incompréhension que vous soulevez dans The New World Climax, les avez-vous vous-même vécues?

B. T. : C'est à force de voyager et de me confronter à des difficultés que j'ai décidé de faire Transit, une œuvre antérieure au New World Climax. Lorsqu'on me voyait dans ces années-là avec mon faciès d'Africain donc de nègre, on se disait que j'étais susceptible de ne pas avoir de papiers. Comme
Entrée de la Bandjoun Station, Bandjoun (Cameroun). (c) D.R.

j'étais souvent interpellé, je me suis dit qu'il fallait faire un travail à partir de cette situation.

Mais cette série n'est pas seulement un résumé des problèmes qu'en tant qu'Africain je rencontrais dans mon travail et dans mes voyages. C'était un travail sur les a priori et les clichés que l'on peut avoir vis-à-vis de tous les marginaux. Ce n'est donc pas juste une réponse au racisme. C'est plutôt un travail universel sur les discriminations et les a priori. Le bénéfice du voyage, de l'exil, du déplacement, c'est d'aller à la rencontre de l'autre. Nous sommes tous en exil sur cette planète. Qu'on soit blanc, noir ou jaune, on devrait pouvoir se déplacer pour des raisons affectives, professionnelles, économiques. Le monde est une richesse, le bien de tous.

$H \& M$ : Depuis vingt ans vous avez beaucoup voyagé. Dans tous ces voyages, que pensez-vous avoir reçu et apporté ?

B. T. : Très jeune déjà, je voulais partir, c'est ce qui me motivait, partir le plus loin possible, pour découvrir autre chose. J'ai ainsi découvert 
d'autres formes d'enseignement artistique à Grenoble, puis à Düsseldorf. En France, j'ai appris de nouvelles techniques, tandis qu'en Allemagne, ce qui a changé pour moi, c'est la notion d'artiste et son rapport avec la vente. J'ai compris que le plus important n'était pas de vendre, mais d'avoir la liberté de travailler ce que l'on aime. Cette liberté est une richesse qui n'a pas de prix. Je travaillais donc sans pour autant vendre, mais j'étais heureux d'être libre, d'être artiste. Ce n'est que par la suite que ma production artistique très engagée a acquis petit à petit une valeur marchande.

$H \& M$ : Vous nous avez dit ce que vous avez reçu en vivant et en travaillant à l'étranger. Mais que pensez-vous avoir apporté ?

B. T. : Ce que j'apporte, c'est d'abord ma culture, mon expérience, mes acquis. Parce que je suis originaire du Cameroun, ma démarche dans la création peut être différente de celle de mes confrères des Pays-Bas, de France ou d'autres pays. Je viens avec une manière différente de penser, de faire, de réfléchir, de fabriquer, de produire. Wilfredo Lam, quand il arrive de Cuba à Paris, vient avec sa manière de dessiner. Picasso arrive avec sa culture de la tauromachie. Je crois qu'il faut célébrer la richesse de l'exil, du voyage.

\section{$H \& M$ : Vous avez installé votre collection d'art} personnelle à l'intérieur de Bandjoun Station. Vous l'avez intitulée Mes amours. Pourquoi avoir décidé de l'ouvrir au public?

B. T. : C'est cette collection qui m'a donné l'envie de créer cet espace. Parce que j'avais constaté que l'art classique africain n'était pas présent sur le continent africain. Les meilleures pièces ont été emportées par les premiers missionnaires, les explorateurs, les colons. Et aujourd'hui, l'art contemporain créé sur le territoire africain ou par les Africains de la diaspora est acheté, certes avec l'accord des créateurs, par les Occidentaux qui ont une idée de l'art plus précise que mes compatriotes. Normal, quand il n'y a pas de volonté poli- tique de constituer des collections d'art contemporain africain dans le pays.

J'ai pensé qu'il fallait construire un lieu pour que les artistes africains et occidentaux puissent montrer leur travail. Il y a une vingtaine d'années, j’ai commencé, lors des rencontres, des biennales ou des grandes expositions collectives, à échanger des œuvres avec des confrères artistes de toutes les nationalités, des collectionneurs, des galeristes à travers le monde. Mon choix est purement subjectif. Il suffit que j'apprécie une œuvre pour demander l'échange, peu importe la technique ou le médium utilisé. Bien sûr, je ne peux m’offrir toutes les œuvres que j'apprécie.

Avec ce système d'échange, j'ai rassemblé une collection que j'expose dans les expositions temporaires et permanente à Bandjoun Station. Au départ, je l'ai conçue pour tout le monde, je ne voulais pas que ce soit réservé à une élite et reproduire le fonctionnement des musées d'art contemporain occidentaux. C'est pourquoi j'ai décidé de lier Bandjoun Station à d'autres pratiques culturelles de la région, pour que les Camerounais puissent s'intéresser à ce qui se fait autour d'eux et à l'extérieur du pays.

H\&M : Vous développez toute une activité agricole pour financer Bandjoun Station et vous avez construit, en plein milieu des plantations, des maisons pour de futures résidences. Pourquoi ce choix ? Comment réagissent les agriculteurs face à ces drôles de maisons couvertes de mosaïques colorées?

B. T. : Ces résidences, qui ne sont pas encore terminées, sont conçues comme des lieux de recueillement, de recherche d'un moment de solitude. L'environnement vert, écologique, moins pollué par des pesticides, me semble propice pour réfléchir, écrire, méditer, se recueillir et se ressourcer.

La deuxième raison est de se rapprocher des personnes qui ne sont pas allées à l'école pour qu'elles puissent profiter de ces peintures et de ces sculptures dans les plantations, dans leurs champs. 
En côtoyant ces univers graphiques, elles auront envie de savoir ce qui se trouve à Bandjoun Station. Quant aux agriculteurs, je pense qu'ils sont contents de travailler dans cet espace. C'est vrai qu'au début, ils trouvaient un peu bizarres voire inquiétants ces dessins, ces scènes oniriques mais, avec le temps, ils ont fini par les apprécier. Comme ces trois gigantesques dents qui s'apprêtent à manger les épis de maïs. Cela a d'abord été perçu comme la figuration d'un acte de sorcellerie, et certains avaient peur de se faire manger par moi comme ces épis. À leurs yeux, j'étais un sorcier. Puis, à force de me voir, la peur est tombée, le malaise s'est estompé. Ces maisons créent dans ces champs tout verts un espace graphique et un dialogue étrange mais plutôt intéressant. Mon but est de faire en sorte que Bandjoun Station ne soit pas réservé à une élite ou à des initiés.

$H \& M$ : Est-ce difficile de faire vivre une telle structure ? Comment la financez-vous, avezvous des soutiens?

B. T. : Jusqu'à aujourd'hui, Bandjoun n'a reçu aucune subvention. Des amis français sont venus et ont décidé de créer l'association des Amis de Bandjoun Station pour assurer le paiement des factures et des salaires des employés du musée.

$H \& M$ : Certains de ces jeunes qui passent par Bandjoun Station vont devenir des artistes professionnels. Quelle est la situation des artistes au Cameroun en terme de formation, d'opportunités?

B. T. : Dans les années 1990, je me suis exilé car j'avais envie de suivre une formation pour ce métier. À mon époque, c'était encore plus difficile. Aujourd'hui, si des écoles existent au Cameroun, il faudrait des maisons de la culture dans les dix provinces pour que les jeunes aient des espaces pour faire de l'art, le montrer, jouer de la musique, du théâtre. Mais ces infrastructures n'existent pas, même pas dans la capitale. Il manque des lieux d'exposition, il n'y a pas de galerie nationale, de musée ouvert au public digne de ce nom, même pas de collections d'artistes camerounais de l'époque moderne à nos jours. Donc, il n'y a pas de repères pour les jeunes. Je pense organiser dans les années à venir une exposition sur la création contemporaine au Cameroun d'aujourd'hui. Il faut parcourir les dix provinces pour découvrir ce qui existe et ce qui va naître, pour constituer un panorama de la création contemporaine au Cameroun. Doual'art fait déjà ce travail à Douala, mais c'est infime par rapport à ce qui devrait être fait dans le pays. Doual'art seul ne peut pas porter la responsabilité de promouvoir l'art contemporain camerounais. L'État aussi doit aussi se mobiliser. 